\title{
Industria de investigación y producción de doctores
}

\author{
Julio Carabaña \\ Departamento de Sociología VI \\ Universidad Complutense \\ carabanya@ccedu.ucm.es
}

El artículo «Sobran o faltan doctores» publicado en el número 7 de Empiria (De Miguel y otros, 2004) trata de muchas cosas además de intentar contestar a la pregunta del título. Lo que yo voy a hacer en esta contribución al debate es en primer lugar centrarme en la cuestión principal, pero sin desdeñar, como añadidura o entretenimiento, fijarme en algunas exageraciones que manifiestamente se han colado en el texto aprovechando que el Pisuerga pasa por Valladolid.

\section{MEJOR QUE SOBREN, SI ES QUE EN VERDAD SOBRAN}

Primero, pues, la cuestión principal. ¿Sobran o faltan doctores?. Evidentemente, estamos ante una de esas cuestiones que, siendo intrínsecamente relativas, tendemos a formular, aunque sólo sea por ahorrar tiempo, de modo absoluto. La formulación correcta es si sobran o falta doctores para algo, por ejemplo, para satisfacer las demandas actuales del mercado, o para satisfacer las necesidades de una situación hipotética. Los autores responden que sobran doctores, aproximadamente unos 1000 al año, para satisfacer las demandas actuales del mercado, pero que faltan para satisfacer las necesidades de una hipotética industria de investigación que se correspondiera con nuestro nivel de desarrollo económico. Lo dicen de un modo quizás algo enrevesado, pero en el fondo es eso lo que dicen: «Sobran porque se producen más de los que absorbe el sistema; faltan porque luego no se dedican a la investigación» (p. 148). Habría sido mejor, desde luego, poner cada cosa en el tiempo verbal apropiado, presente y actual para el exceso, futuro e hipotético para el defecto. De hecho sobran doctores, aunque faltarían si tuviéramos una investigación como es debido. 
Si el artículo se limitara a sacar esta conclusión habría verdaderamente poco que debatir. Es indiscutible que faltarían doctores si el nivel de I+D en España fuera el corresponde a su desarrollo económico, y seguramente muchos más si nuestro nivel de investigación fuera equivalente al de Estados Unidos. No lo es, y cuantos más sobran de hecho, más claro está que nuestro desfase no se debe a su ausencia. El debate, por consiguiente, tendría que reducirse a la cuestión de si están bien estimados esos 1000 doctores que salen cada año de las universidades y no encuentran acomodo en el sistema como tales. No parece realmente una cuestión muy grave, en primer lugar por el escaso número de los afectados: 1000 doctores no son al cabo mucho cuando tenemos decenas de miles de licenciados que también salen cada año del sistema y no encuentran trabajo en sus respectivos campos. Y, en segundo lugar, la cuestión tampoco parece grave porque muy probablemente los doctores encuentran trabajos de licenciado con mucha mayor facilidad que éstos, de tal modo que el tiempo y esfuerzo invertidos en realizar la tesis no quedan sin recompensa. De todas formas, hay que decir que el modo como se estima este exceso de doctores es bastante objetable. El procedimiento (tabla 3, p.136) consiste en hallar la diferencia entre el número de nuevos doctores y el número de nuevos profesores de universidad. Es evidente que por este procedimiento se están dejando fuera todos aquellos doctores, ciertamente de difícil localización, que encuentran trabajo como investigadores o en cualesquiera otros puestos para los cuales su condición de doctor ha tenido alguna relevancia. La conclusión, por lo tanto, debería ser más bien la siguiente: quizás haya algunos estudiantes, 1000 como mucho, que aún sacando provecho laboral de sus estudios de doctorado no se colocan en puestos para los que se exige el título de doctor, como profesores de Universidad e investigadores.

Todavía puede decirse más. No sólo es este un problema menor, sino que lo es solamente desde el punto de vista de la economía, y más concretamente de la economía individual. Ahora bien, el punto de vista de la economía individual no es el único punto de vista que importa, así que antes de pasar a las recomendaciones prácticas hay que considerar otros puntos de vista. Por ejemplo, el punto de vista académico, o del doctorado como curiosidad y cultivo del saber. Teniendo en cuenta que en la decisión de realizar la tesis doctoral hay en muchísimos casos una enorme dosis de actitud 'consumista' y muy poco de actitud 'inversora', por decirlo en los términos prosaicos de la teoría del capital humano, el fenómeno no debería parecer alarmante sino, por el contrario, halagüeño. No estaríamos ante un problema, sino ante la manifestación de la vocación intelectual y científica, independiente del interés crematístico, de una minoría de nuestros licenciados universitarios. El amor al saber, el aprender por aprender, es la actitud que muchos predican como el espíritu propio de la Universidad. No debería sorprendernos, y menos preocuparnos, la existencia de una exigua minoría de unos mil estudiantes que, insatisfecha con lo estudiado y aprendido en la licenciatura, mantiene este espíritu 'filosófico' - de amor al saber - durante algunos años más. Si miramos la cosa desde este punto de vista, no podemos acusar 
a los estudiantes de perder su tiempo en la adquisición de saberes que no les van a ser laboralmente útiles, ni tampoco tomar medidas para dificultarles o impedirles tal derroche.

Podemos, en cambio, acusarlos de utilizar para su simple deleite recursos públicos, y, si no impedírselo, sí obligarlos a pagar el coste íntegro de los estudios. Si estudian por amor al arte y disfrutan con ello, que lo paguen. Pero hay todavía un argumento para subvencionar los estudios de doctorado, como lo hay para subvencionar la enseñanza en general, que es el argumento de las externalidades. Junto al punto de vista económico de los beneficios individuales, está el punto de vista, no menos económico, de los beneficios colectivos. Beneficios que en el caso del doctorado son incluso un producto tangible, las tesis doctorales en las que se plasma la contribución de los doctores a la investigación. ¿Valen estas tesis lo que los contribuyentes pagan por ellas?. Es imposible responder a esta pregunta de modo absoluto, pero sí en relación a la investigación realizada por profesionales. Y la respuesta creo que debe ser la afirmativa. Quizás la investigación desarrollada por los doctorandos no alcance la calidad de la investigación realizada por los doctores que investigan en el tiempo que les deja libre la enseñanza universitaria o a tiempo completo en los Organismos Públicos o instituciones privadas de investigación; pero el beneficio neto es muy probablemente superior por la simple razón de que los doctorandos trabajan gratis, o a lo sumo mantenidos por modestas becas cuyo importe apenas supera el salario mínimo. Son todavía 'estudiantes profesionales' antes que 'trabajadores científicos'. Las becas, en efecto, son muy inferiores a lo que los licenciados mejores pueden obtener en otros empleos e incluso a lo que cobran los investigadores de plantilla (Larraga, 2003).

Por cerrar esta cuestión central, podemos concordar con de Miguel y sus coautores en que sobran doctores sólo desde el punto de vista de la inversión individual en capital humano. Pero no si adoptamos el punto de vista de la inversión social o el del disfrute individual. Hacer el doctorado sería en todo caso una actividad altruista, en la que el actor se perjudica pero la colectividad se beneficia. Cualesquiera medidas políticas que se tomaran para reducir el número de doctores quizás mejorarían la rentabilidad individual de los estudios de doctorado, pero al coste de coartar la libertad de las personas y de renunciar a los beneficios sociales de su actividad investigadora.

\section{¿MUCHOS ESTUDIANTES Y POCOS GRADUADOS?}

De Miguel y sus colegas tratan además de la cuestión central muchas cuestiones secundarias o laterales. A la que más importancia dan es a la desproporción entre el número de doctores y el número de estudiantes de doctorado. Su opinión es que se trata de un despilfarro: «Dado que, a pesar de ser un sistema eminentemente público, España no regula ni planifica la producción de doctores, 
la falta de coordinación y el malgasto de recursos es considerable. La autorregulación - sin ningún tipo de planificación central y autonómica - produce que cada año haya más personas empezando el doctorado y proporcionalmente menos personas terminando. La productividad es de alrededor de 1 de cada 5 estudiantes. El coste material (en recursos universitarios) y personal (los propios estudiantes de doctorado que nunca logran el título) es enorme. La tensión entre gasto y calidad en las universidades españolas es evidente». $Y$ luego: «El problema fundamental es averiguar por qué cuatro de cada cinco estudiantes de doctorado nunca termina los estudios que deseaban realizar. Quizás la pregunta sea al revés: ¿por qué se matricularon en doctorado si realmente no querían ser doctores?. Las hipótesis son múltiples. Una razón es el mercado de trabajo...» ... etc, etc. Siguen con el asunto nuestros autores hasta ocupar con él cerca de la mitad del espacio dedicado a las conclusiones.

Debemos preguntarnos ante todo si los hechos son así. La respuesta es que no. Todo indica que nos encontramos ante un fenómeno harto frecuente en los estudios sociales: el deficiente establecimiento de los hechos (Merton, 1987). Este descuido es una plaga en los estudios llamados cuantitativos, en muchos de los cuales con tal de aplicar métodos estadísticos complejos parece que no importa mezclar peras con manzanas. ¿Cómo se ha llegado a la conclusión de que hay en España muchos estudiantes de doctorado y pocos doctores?.

Se ha llegado del modo siguiente: «La población estudiantil anual que puede empezar estudios de doctorado es de aproximadamente 120.000 estudiantes. Al año siguiente hay matriculados en doctorado unos 64.000 estudiantes. Pero la matrícula tiene que dividirse entre los dos años obligatorios de formación doctoral, lo que supone unos 32.000 estudiantes de doctorado. Estos datos, si son fiables, sugieren que al menos uno de cada cuatro estudiantes $(27 \%)$ que se licencian siguen estudios de doctorado, lo que intuitivamente parece una cantidad excesiva».

Efectivamente, la intuición acierta: es una cantidad excesiva. Los 64.000 estudiantes de doctorado han de dividirse, no por los dos años obligatorios de formación doctoral, sino por los años que se tarda en terminar la tesis. Pues durante todos esos años los alumnos cuentan como estudiantes de doctorado y pagan una matrícula de mantenimiento de la inscripción de la tesis. No deja de resultar un poco excesivo que la primera recomendación práctica de los autores se dirija al INE y sea la de mejorar los datos. Es verdad que los datos estadísticos siempre pueden mejorarse. Pero si los datos son tan malos, es temerario construir sobre ellos tantas páginas de prosa tan categorica. A la desproporción entre doctorandos y doctores los autores le dedican un amplio espacio, primero intentando explicar el fenómeno y luego proponiendo remedios para arreglarlo. Se habrían ahorrado el esfuerzo de haber establecido previamente los hechos, para lo cual habría bastado con hacer caso a su cotidiana experiencia como profesores universitarios. Más allá de esta experiencia diaria podrían haber ido teniendo en cuenta otras fuentes de datos distintas de la Estadística de la Ense- 
ñanza en España, como por ejemplo el Censo o la Encuesta de Población Activa. Por ejemplo, la EPA del segundo trimestre del año 2000, dedicada por cierto con no mucha fortuna al estudio de la inserción profesional de los jóvenes, proporciona las siguientes cifras (en porcentajes de la población de cada edad):

\begin{tabular}{lcc}
\hline Años de nacimiento & $\begin{array}{l}\text { Licenciados y } \\
\text { estudiantes } \\
\text { de licenciatura }\end{array}$ & $\begin{array}{l}\text { Doctores } \\
\text { y estudiantes } \\
\text { de licenciatura }\end{array}$ \\
\hline $1961-65$ & $9 \%$ & $0,5 \%$ \\
$1966-70$ & $10 \%$ & $0,6 \%$ \\
$1971-75$ & $20 \%$ & $0,7 \%$ \\
\hline
\end{tabular}

Es verdad que los datos no son buenos. La EPA obtiene una muestra muy pequeña de doctores (unos 15 de cada edad) y podría favorecer nuestros cálculos preguntando tanto si se han realizado estudios de doctorado como si se han terminado, para de ese modo permitirnos calcular las tasas de abandono. En lugar de esto pregunta por los estudios terminados y por los estudios en curso, de lo cual resulta que mientras las cifras de 1961-65 son en su mayor parte de titulados, las de 1971-75 son en buena parte de estudiantes, muchos de los cuales no terminarán nunca. Aún así, los datos bastan para estimar con razonable certeza que de los nacidos en los sesenta se doctoró uno de cada veinte licenciados y que esta ratio no ha aumentado, sino quizás disminuido hasta uno de cada treinta entre los nacidos en los setenta. Además, debe considerarse que la mayor parte de los nacidos entre 1961 y 1965 han acabado los estudios en el año 2000. La ratio $0,6 / 10$ es, por tanto, es entre doctores y licenciados. Entre los nacidos en 1971-75, en cambio, la mayor parte son todavía estudiantes, sobre todo en doctorado; la ratio $0,7 / 20$, es entre estudiantes de doctorado y estudiantes/licenciados universitarios. No hay según la EPA nada parecido a la enorme inflación de estudiantes de doctorado que tanto alarma a nuestros autores.

Pero no se crea que de resultar los hechos ciertos el diagnóstico habría sido sin más acertado. Supongamos que, en efecto, la productividad fuera de un doctor por cada cinco estudiantes de doctorado. Ante de dictaminar que este hecho es un problema que requiere solución, es preciso analizar las ventajas y desventajas, los costes y beneficios de esta situación, y comparar su balance con el que se espera tras la intervención política que se propone. El primer balance parece obvio: disminuirían los costes si sólo empezara el que termina, aumentarían los beneficios si terminaran todos los que empiezan. Pero este balance está construido sobre el supuesto de costes iguales para todos los que empiezan y beneficios sólo para los que terminan, un supuesto que bien puede ser inadecuado. Hay otro supuesto, más plausible, el de proporcionalidad entre costes, beneficios y tiempo. Quien se limita a hacer los cursos de doctorado sin terminar la tesis no está perdiendo el tiempo si, como es de esperar, está aprendiendo. Máxime cuando el Gobierno, siguiendo avant la lettre las propuestas de los autores (p. 153), 
ya otorga el DEA (Diploma de Estudios Avanzados) a quienes terminan los dos años de doctorado. Incluso aunque el primer balance fuera el correcto y los estudios de doctorado no sirvieran de nada sin la tesis doctoral, todavía podría justificarse el presunto exceso de estudiantes como un proceso de selección cercano al óptimo. En efecto, los procesos de selección in actu exercitu, a diferencia de la selección por indicadores, tienen fiabilidad perfecta. Al no cometer errores, tampoco generan dudas sobre la legitimidad del proceso. Nadie puede pretextar que no se le dio la oportunidad de ser doctor sobre la base de los resultados de una prueba imperfecta. En cuanto al segundo balance, la relación costes-beneficios de la situación que los autores consideran mejor, es imposible llevarlo a cabo mientras no propongan algo más concreto que 'una organización racional de los recursos'. En suma, que ni aún si los datos estuvieran bien interpretados se nos han dado razones para considerar que estamos ante un problema, y ni aún si estuviéramos ante un problema tienen visos de mejorar la situación las vagas políticas que los autores proponen.

\section{DESIGUALDADES, PERO NO DISCRIMINACIONES}

La desproporción entre estudiantes y doctores es sólo uno de los muchos asuntos que de Miguel y sus colegas califican de problema sin documentación empírica ni justificación normativa. Por ejemplo, en la p. 117 se declara en decadencia a la universidad europea, y en la p. 118 'se observan diez problemas' en ella. Salta a la vista que la mayor parte de sus descripciones carece de base empírica, de justificación política o de ambas. Por ejemplo: «falta una estratificación de universidades que suponga una especialización de funciones, así como una dedicación diferente a la formación de docentes e investigadores», diagnóstico que se completa en la conclusión con la propuesta de que «los doctores se produzcan en unos pocos centros de excelencia que luego empiecen a trabajar en otras instituciones». ¿Como se justifican diagnóstico y remedio?. Pues porque parece que tal jerarquía ocurre en Estados Unidos. Las ratios norteamericanas son la referencia habitual, por ejemplo para los investigadores en relación a la población. Son una referencia arbitraria, por mucho que la investigación sea una rama de actividad menos boyante en España que en Estados Unidos.

Probablemente el más bizarro de todos estos pseudo problemas es la desigualdad entre Comunidades autónomas. La información empírica es que en el conjunto de España se producen cuatro doctores por cada mil estudiantes universitarios, en Navarra 9,8, en Madrid 5,8, en La Rioja 0,4, en Castilla la Mancha 0,8 . Es, desde luego, legítimo, preguntarse porqué. También es legítimo explorar esta pregunta mediante correlaciones y llegar a conclusiones como que estas diferencias no se deben a la proporción de mujeres profesoras o como que la variable clave es el gasto por estudiante. Pero no tiene mucho sentido convertir la descripción en diagnóstico y la situación en problema asegurando que 
«no parece que eso preocupe mucho al gobierno central ni a los autonómicos, ni existen planes de convergencia en el tema del doctorado». Sobre todo cuando se apunta en una nota que «no existe un consenso sobre si las CCAA deberían producir el mismo número (o parecido) de doctores)». ¿En qué quedamos?.

En esta línea de arbitrariedad normativa resulta particularmente irritante la permanente confusión de desigualdad con discriminación cuando de hombres y mujeres se trata. He aquí algunos párrafos para que sirvan de muestra: (1) «En España se producen progresos, como la extensión del número de estudiantes (cuya tasa actual está estancada) o el avance de las tasas de feminización del alumnado (que alcanza ya a 120 mujeres por cada cien varones). Pero la feminización no ha llegado en algunas áreas (como ingeniería), ni tampoco al profesorado (que sólo cuenta con el $34 \%$ de mujeres). En los estratos de catedráticos, cargos y poder universitario la dominación masculina es rampante» (p.119). (2) En Estados Unidos faltan doctores, sobre todo nacionales, no se les paga bien y, además, «es necesario que el proceso de feminización continúe y se complete. Entre los doctores nuevos hay un $44 \%$ de mujeres, pero dado que hay más mujeres estudiando en la Universidad, ese porcentaje debería elevarse por lo menos hasta el $50 \%$. El esfuerzo tiene que ser mucho mayor en ingeniería, donde las mujeres son únicamente el 17\%, o en Ciencias, 25\%» (p. 127). (3) En España, en cambio, 'no puede hablarse ya de discriminación de la mujer en la Universidad, ni siquiera en el doctorado...No existe ya discriminación y es muy posible que en el futuro la situación sea la inversa (falta de varones). En la tabla 5 puede observarse que hay todavía una discriminación clara en Ingeniería. Ingeniería es todavía un feudo masculino...». Pueden multiplicarse ad libitum las citas, pero creo que las anteriores bastan para atestiguar que los autores suponen que toda diferencia favorable a los hombres es discriminatoria y debe ser corregida.

Es un supuesto claramente falaz. Las diferencias de resultados entre dos grupos o categorías de individuos indican discriminación en los procesos únicamente cuando los grupos o categorías tienen 1. las mismas cualidades relevantes y 2. la misma motivación. Estas condiciones son muy fuertes, pues es muy difícil determinar que en efecto, dos categorías tienen idénticas cualidades y motivación. Podría parecer que las cualidades relevantes deben suponerse iguales cuando las categorías se distinguen por cualidades obviamente irrelevantes, como la altura, el peso corporal, el color de la piel, el sexo o la belleza parecen serlo para el acceso a la Universidad. Podría entonces decirse que las desigualdades asociadas a ellas se deben a discriminación en los procesos. La investigación ha descubierto, sin embargo, insospechadas correlaciones entre rasgos en apariencia independientes, por ejemplo altura e inteligencia, suficientes para explicar que la diferencia de doctores entre digamos las personas altas y las bajas no se debe a discriminación. En general, la igualdad de cualidades y de motivación entre dos grupos, incluso si lo que los distingue es una cualidad aparentemente irrelevante, es tan difícil de establecer que es temerario interpretar las diferencias entre ellos como discriminación si no se dispone de alguna eviden- 
cia directa. Así las cosas, es temerario hablar de discriminación por sexo en la enseñanza, dado que, por un lado, las diferencia en aptitudes y motivación entre hombres y mujeres están bien establecidas, y por otro es difícil encontrar evidencia de procesos discriminatorios en la escuela. De hecho, lo que los estudios muestran es el papel decisivo de las motivaciones: a medida que estas se han hecho favorables a los estudios, los resultados académicos de las mujeres han superado a los de los hombres, poniendo en tesitura difícil a los acusadores del sistema educativo. Tal como dicen nuestros autores: «No existe ya discriminación y es muy posible que en el futuro la situación sea la inversa (falta de varones)».

La desigualdad de motivación es particularmente delicada, pues siempre hay quien se atreve a decir que tiene un origen social y que debería ser corregida. Hay aquí una aberración triple, moral, sociológica y política. Moral porque fuerza la libertad de los individuos para conseguir un resultado global predeterminado. En el dilema entre libertad e igualdad, se decanta por la igualdad, aún a conciencia de que habrá de violentar la libertad, condenada por estar socialmente influida. Sociológica porque es producto de un malentendido elemental: confunde tasas y cuotas, porcentajes de origen y de destino. Tomemos el hecho de que sólo el $25 \%$ de los ingenieros son mujeres. La aberracion se produce por una variación aparentemente inocua en la formulación del hecho: pasamos de decir que la ingeniería es una carrera masculina a entender que la masculinidad es muy ingeniera. Los hombres son buenos para la mecánica, las mujeres no. No es lo mismo, sin embargo. De hecho, ni las mujeres ni los hombres están muy motivados para la ingeniería. Tres de cada cien varones decide estudiar ingeniería, frente a una de cada cien mujeres. La mayoría de las personas, hombres y mujeres, eligen estudiar cosas distintas de la ingeniería. Lo correcto es decir que la vocación por la ingeniería es un fenómeno raro, aunque se da con frecuencia algo mayor entre los hombres que entre las mujeres. Pasa lo mismo con muchos otros comportamientos. Por ejemplo, el robo a mano armada es un comportamiento harto infrecuente, aunque se da casi en exclusiva entre los hombres. Nadie dice por ello que los hombres son más ladrones que las mujeres. Ocurre que 'masculino' y 'femenino' se dicen de muchas maneras. En un extremo hay comportamientos universales y diferenciales, o casi, como los ligados directamente al sexo; de estos, por ejemplo, dar el pecho a las crías, es de los que propiamente se dice que son masculinos o femeninos. En el otro extremo tenemos comportamientos singulares y comunes a ambos sexos, que simplemente son más frecuentes en un sexo que en otro, pero que no por ello merecen el adjetivo masculino o femenino, pues esa mayor frecuencia es contingente o accidental. Es en parte lo que se ha querido diferenciar con la distinción entre sexo y género, aunque equivocadamente, pues no puede equipararse el sexo a lo permanente y lo social a lo arbitrario; hay comportamientos 'sexuales' fácilmente alterables por costumbres sumamente resistentes al cambio, como atestigua, por ejemplo, la pauta europea de matrimonio. En fin, el hecho de que un porcentaje mínimo de hombres y un porcentaje asimismo mínimo, pero algo mayor o menor, de mujeres tenga un comportamiento determinado, no autoriza a afirmar 
que sea femenino o masculino, ni a buscar las causas de la diferencia de frecuencia en rasgos propios de cada sexo, por mucho que nos incline a ello el hecho de que la mayor parte de sus practicantes sean machos o hembras.

La aberración política es consecuencia directa de las otras dos. Si aceptamos la falsa formulación de que los hombres son más ingenieros que las mujeres y la equivocada premisa moral de que deberían ser iguales entonces concluiremos que es necesario motivar más a las mujeres para que sean ingenieras, o a los hombres para que no sean delincuentes. En ambos casos la igualdad es una meta moral errónea. En el de la delincuencia la meta correcta es que nadie sea delincuente, en el caso de la elección de estudios la meta correcta es que cada cual elija libremente. Pero suponiendo que aceptáramos la igualdad como meta, ¿qué podríamos hacer para conseguirla?. En el caso de los ingenieros se trataría de convencer a un dos por ciento de universitarias de que eligiera ingeniería. El mínimo esfuerzo exigiría, no fomentar el amor a la ingeniería entre las mujeres en general, sino convencer a las más inclinadas. También podríamos convencer de que no eligieran ingeniería a los hombres menos inclinados, o de que se hicieran atracadoras a las mujeres con mayor propensión a la violencia. En ambos casos, ellas/ellos habrían sacrificado sus preferencias individuales; se supone que en conjunto las mujeres estarían contentas, pues habrían logrado el objetivo de igualarse a los hombres. Sería de desear por muchas razones, no solamente de virtud ciudadana, que fueran los predicadores de la igualdad de resultados estadísticos los que se desprendieran de las influencias sociales que condicionan sus opciones, cambiaran sus preferencias, se sacrificaran por la igualdad estadística y estudiaran ingeniería o se dedicaran a la delincuencia.

\section{¿SON BUENOS NUESTROS DOCTORES?.}

Como esto pretende ser un debate e ir más allá de una crítica que por ahora pudiera antojarse meramente negativa, voy a resumir algunas hipótesis sobre el número de doctores y más en general sobre la situación de la investigación en España que he desarrollado más ampliamente en otro lugar. A fines del año pasado, la FECYT organizó un seminario en el cual se trataba de esbozar escenarios para la investigación a un par de décadas vista. Me encargaron de imaginar como evolucionarían aquellos aspectos del sistema de enseñanza más relevantes para la investigación. Puesto a la faena, no me sorprendió encontrar que el casi único rasgo relevante para la investigación es, desde el punto de vista de la oferta, el nivel de la elite de los licenciados. Y me deprimió encontrar asimismo fuertes indicaciones de que esta elite es en España muy inferior a la de muchos países desarrollados, tanto europeos como americanos, y no digamos Japón y Corea.

¿Cómo llegué a este resultado? A partir del informe PISA, elaborado por la OCDE. Este informe permite comparar la habilidad lectora, matemática y científica de los alumnos de 15 años de más de 30 países de la OCDE, entre ellos España. Gracias a este Informe sabemos que en lo que se refiere a resultados glo- 
bales la Enseñanza Básica española es aceptablemente eficaz y sumamente eficiente. Es aceptable en eficacia porque los alumnos españoles desarrollan su capacidad cognitiva aproximadamente igual que los alumnos de los otros países de la OCDE (OCDE, 2001). Según el informe PISA, los alumnos españoles alcanzan en lectura una puntuación de 493, en Matemáticas de 476 y en Ciencias de 491, que hay que comparar con una media de 500 del conjunto de los países. Aunque la prensa y los políticos destacaron que España quedaba por debajo de la media, la diferencia, como correctamente han leído Pérez-Díaz y Rodríguez ((2003:447), es muy pequeña, y tanto podía España estar en el puesto $18^{\circ}$ como en el $15^{\circ}$ o en el $20^{\circ}$, pues su media no puede en realidad distinguirse de la de Estados Unidos (504), Dinamarca (497), Suiza (494), Italia (487) y Alemania (484), y apenas es inferior a la de Francia (505). En realidad, el Informe PISA muestra que los alumnos de todos los países de la OCDE (menos México) tienen a los quince años capacidades cognitivas extraordinariamente semejantes, planteando de este modo un difícil desafío a cualquiera que pretenda argumentar a favor o en contra de un determinado modelo de sistema educativo o método de enseñanza.

Otra importantísima conclusión que puede sacarse del informa PISA es que el gasto no influye. Si consideramos sólo los países desarrollados hay una debilísima relación estadística entre gasto y resultados, tan débil que desaconseja todo intento de mejora de los segundos mediante el aumento del primero. Resulta de ello que el sistema español de enseñanza está entre los primeros eficiencia, superado sólo por algunos países de la Europa Oriental, como Chequia, Hungría o Polonia, que gastan todavía menos y obtienen resultados semejantes, y por Corea e Irlanda, que obtienen mejores resultados con parecido gasto. Aunque España dobló durante los primeros noventa el gasto por alumno en la enseñanza no universitaria, en parte por las políticas socialistas y sobre todo por la disminución de alumnos, todavía está entre los países de Europa Occidental que menos gastan.

La tercera conclusión que puede extraerse del Informe PISA es que la desigualdad de aprendizaje de los alumnos españoles de 15 años está entre las menores del mundo, tanto considerados individualmente como agrupados en categorías. Tan gran igualdad se debe por un lado a que, contra lo que hemos creído durante mucho tiempo, tenemos pocos alumnos con resultados bajos. Bajo nivel 1, un umbral arbitrario, pero objetivo y único para todos los países, situado en 335 puntos en la escala de lectura, está el 5,7\% de los alumnos de la OCDE, pero sólo el 3,5\% de nuestros alumnos de 15 años; un porcentaje semejante a los de Bélgica, Dinamarca, Finlandia, Francia, Islandia, Irlanda, Italia, Holanda, Suecia y el Reino Unido. Están, en cambio, peor que la media, además de Brasil, México o Rusia, países como Noruega, Polonia, Portugal, Grecia, Alemania y Estados Unidos.

Pero tanta igualdad, y aquí viene el punto que nos interesa directamente, se debe también a que tenemos pocos alumnos con resultados altos. Nuestra Enseñanza Básica está entre las que menos alumnos destacados produce. Tal nivel lo fija arbitrariamente el Informe PISA en los 626 puntos (nivel 5). La agrupación de los países según su proporción de alumnos de nivel alto (como puede verse en la tabla 1) es la siguiente: 
Menos de $1 \%$ de alumnos de nivel 5: Brasil y México.

En torno al 3\% de alumnos de nivel 5: España, Portugal, Italia, Grecia, Rusia, Polonia, Letonia, Hungría, Corea.

En torno al 10\%: Suecia, Noruega, Dinamarca, República Checa, Austria, Alemania, Suiza, Francia, Japón, Estados Unidos.

En torno al 15\%: Finlandia, Holanda, Bélgica, Reino Unido, Irlanda, Australia, Nueva Zelanda, Canadá.

Los alumnos que se sitúan a nivel 5 son aquellos que son capaces de realizar las tareas de lectura más sofisticadas, como relacionar información compleja en textos largos o poco familiares, mostrar una comprensión profunda y matizada del texto, distanciarse de él, evaluar de modo crítico su forma y su contenido o incluso adoptar un punto de vista contraintuitivo. Parece evidente que de los alumnos de este nivel depende el desarrollo de la investigación, la ciencia, la técnica, el arte y la industria. Y parece evidente también que en España son muy pocos. ¿Cuántos alumnos españoles hay por encima del percentil 95 de la OCDE, 650 puntos?. Pues el 1,15\% de alumnos en Matemáticas y el 2,6\% de los alumnos de Ciencias. ¿Y por encima del percentil 95 de Canadá en Matemáticas, que es de 668 ?. Pues $0,8 \%$. ¿Y por encima del percentil 95 de Canadá en Ciencias, que es de 670 puntos. Pues $1,7 \%$. Por último, donde hay $5 \%$ de japoneses (por encima de 688 en Matemáticas y en Ciencias) sólo hay 0,3\% de españoles en Matemáticas y $0,9 \%$ en Ciencias.

TABLA 1 .

Porcentajes de alumnos de 15 años por países y percentiles en la prueba pisa 2000 de lectura.

\begin{tabular}{|c|c|c|c|c|c|}
\hline \% horizontal & $\begin{array}{c}\text { BAJO } \\
5 \%\end{array}$ & $\begin{array}{c}\text { MEDIO } \\
90 \%\end{array}$ & $\begin{array}{c}\text { ALTO } \\
5 \%\end{array}$ & Total & $\%$ vertical \\
\hline ALEMANIA & $\begin{array}{l}285 \\
\end{array} 5,6$ & $\begin{array}{l}4553 \\
\\
\end{array}$ & 235 & 5073 & 2,9 \\
\hline AUSTRALIA & $\begin{array}{ll}78 & \\
& 3,4\end{array}$ & $\begin{array}{l}4476 \quad 86,5 \\
\end{array}$ & 552 & 5176 & 3 \\
\hline AUSTRIA & 192 & 4417 & 136 & 4745 & 2,7 \\
\hline BELGIUM & 5,2 & 89,3 & 5,5 & 6670 & 3,8 \\
\hline BRAZIL & $\begin{array}{ll}1257 & \\
& 25,7\end{array}$ & 74,1 & 0,2 & 4893 & 2,8 \\
\hline
\end{tabular}


JULIO CARABAÑA

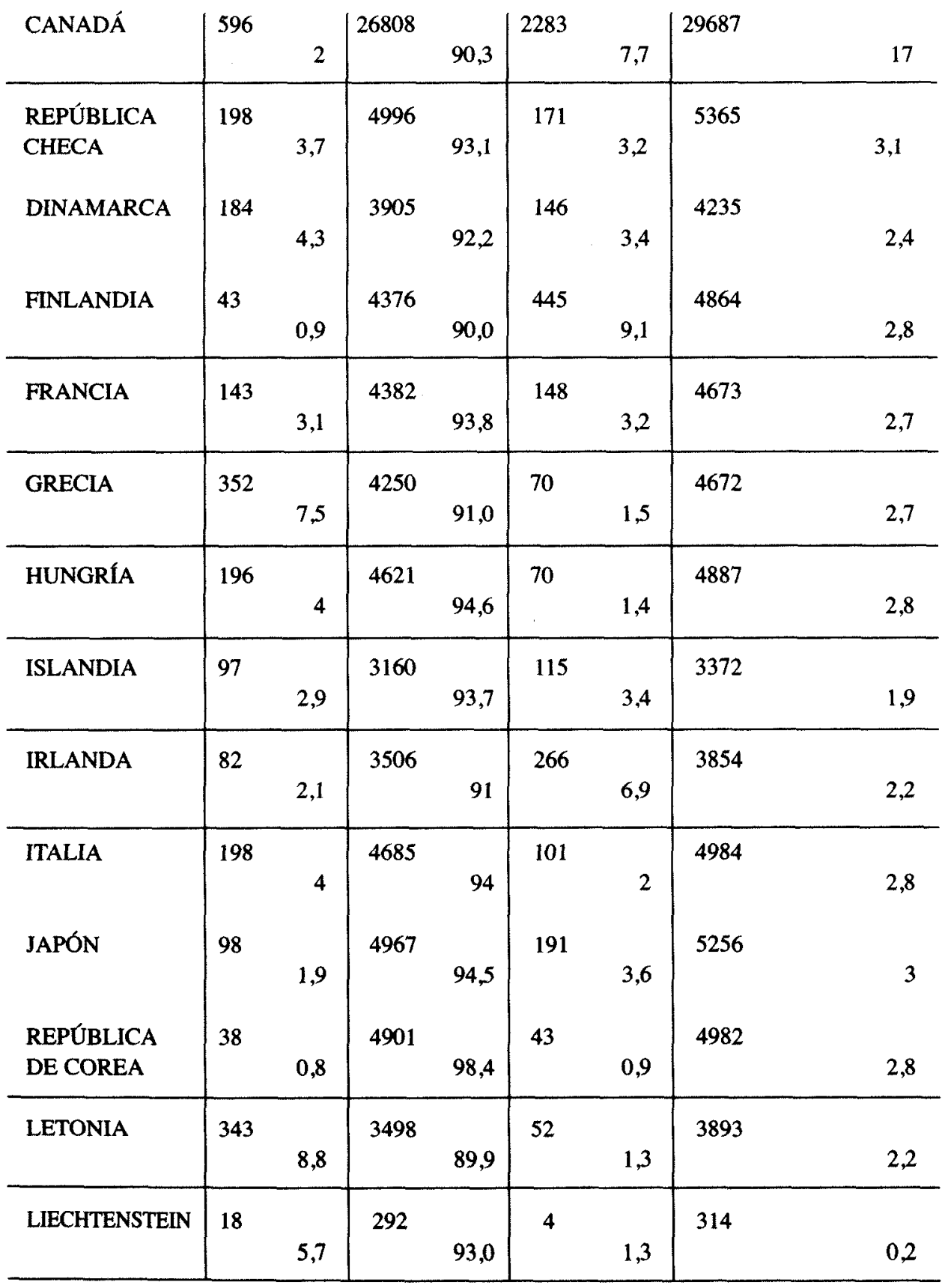


LA INTRODUCCION EN FRANCIA DE LOS MÉTODOS DE SONDEO ALEATORIO

\begin{tabular}{|c|c|c|c|c|c|}
\hline LUXEMBURGO & $\begin{array}{ll}377 & \\
& 10,7\end{array}$ & $\begin{array}{l}3145 \quad 89,1 \\
\end{array}$ & $\begin{array}{ll}6 & \\
& 0,2\end{array}$ & 3528 & 2 \\
\hline MÉXICO & $\begin{array}{ll}507 & \\
& 11\end{array}$ & $\begin{array}{ll}4086 & \\
& 88,8\end{array}$ & 7 & 4600 & 2,6 \\
\hline HOLANDA & 1,3 & $\begin{array}{l}2283 \\
91,2\end{array}$ & 187 & 2503 & 1,4 \\
\hline $\begin{array}{l}\text { NUEVA } \\
\text { ZEALANDA }\end{array}$ & 142 & 85,5 & 389 & 3667 & 2,1 \\
\hline NORUEGA & 205 & 3731 & 211 & 4147 & 2,4 \\
\hline POLONIA & 7,9 & 90,9 & 1,3 & 3654 & 2,1 \\
\hline PORTUGAL & 302 & $\begin{array}{l}4221 \\
92,1\end{array}$ & 1,4 & 4585 & 2,6 \\
\hline $\begin{array}{l}\text { FEDERACIÓN } \\
\text { RUSA }\end{array}$ & 6,2 & 92,6 & 1,2 & 6701 & 3,8 \\
\hline ESPAÑA & 2,7 & 96,2 & 1,2 & 6214 & 3,6 \\
\hline SUECIA & 96 & $\begin{array}{l}4104 \quad 92,9\end{array}$ & 216 & 4416 & 2,5 \\
\hline SUIZA & 269 & $\begin{array}{l}5602 \quad \\
\\
91,8\end{array}$ & 229 & 6100 & 3,5 \\
\hline $\begin{array}{l}\text { REINO } \\
\text { UNIDO }\end{array}$ & 2,8 & 88,2 & 8,9 & 9340 & 5,3 \\
\hline $\begin{array}{l}\text { ESTADOS } \\
\text { UNIDOS }\end{array}$ & 204 & 3429 & 213 & 3846 & 2,2 \\
\hline Total & $\begin{array}{ll}8114 & \\
& 4,6\end{array}$ & $\begin{array}{ll}158857 & \\
& 90,8\end{array}$ & 7925 & 174896 & 100,0 \\
\hline
\end{tabular}

Fuente: Explotacion propia, Fichero PISA 2000. www.pisa.oecd.org 
Estos son los resultados de los alumnos de quince años en el año 2000 . Recuérdese que en virtud de la primera conclusión (vide supra) es muy difícil (sólo difícil, no imposible) atribuirlos a rasgos organizativos de los sistemas de enseñanza, y en virtud de la segunda es también poco probable que se pueda hacer mucho con dinero. Tenemos, pues, sólidos fundamentos para realizar algunas estimaciones sobre la cantidad y el nivel de nuestros investigadores en activo. Si los resultados del informe PISA son extensibles a toda la población, para cualesquiera tareas que exijan las competencias propias del percentil 95 (655) habría cinco individuos de la OCDE por cada español en Matemáticas y dos por cada español en Ciencias. Si las competencias necesarias fueran de nivel aún más alto (688) habría diecisiete japoneses por cada español en matemáticas y cinco por cada español en Ciencias.

Si en Estados Unidos hay tres veces más alumnos de nivel alto que en España pero menor proporción de doctores, es claro que los doctores de Estados Unidos tienen un nivel mucho más alto que los doctores españoles. En España se conceden menos de 2000 becas de investigación nuevas cada año, es decir, aproximadamente el $0,5 \%$ de una generación de 400,000 efectivos. Suponiendo que obtienen beca los mejores alumnos, tendríamos el corte en los 675 puntos de la escala PISA. Por encima de ese punto, tienen el 5\% de su población Estados Unidos y muchos países de la OCDE. Creo que la conclusión a estas alturas es clara: si nuestra élite escolar no mejora en el Bachillerato y en la Universidad (condición que no hay motivos para afirmar), cuando llaga al doctorado es realmente una elite bastante vulgar.

¿Qué relación tiene esto con la investigación?. Resulta sugerente comparar las proporciones de estudiantes brillantes de la tabla 1 con los indicadores de esfuerzo dedicado a la investigación en los diversos países. Por ejemplo, el personal de I+D por cada mil trabajadores es más de diez en Japón, Francia y Alemania, menos del 5 en Grecia, Portugal, Italia o España, e intermedio en la mayor parte de países europeos. Los porcentajes del PIB gastados en investigación guardan una relación parecida. Idea: cuando hay una buena cantera de investigadores, la investigación se desarrolla en una dinámica de círculo virtuoso porque ellos atraen la financiación. La razón principal por la cual en un país como España hay poca investigación no es falta de inversión, sino la falta de una elite académica de alto nivel de la cual extraer investigadores competitivos que consigan dinero.

La cuestión se agrava si, además de mala cantera, los procedimientos de selección son poco fiables e incluso a veces, como ocurre en la Universidad con los profesores asociados, dan como resultado una selección adversa. Por un lado acaban pasando por investigadores quienes han sido seleccionados inicialmente para resolver problemas inmediatos de docencia como profesores asociados $(\mathrm{Cf}$. Carabaña, 1999; 2002). Por otro lado, los becarios de investigación se refugian en cuanto pueden en un puesto de profesor que asegura el inicio de una carrera docente. Un indicio de esta segunda selección adversa puede encontrarse Fer- 
nández Esquinas (2002) en cuyas tablas 5.2 y 5.10 puede verse que aproximadamente la mitad de los becarios de investigación de la Universidad, tanto en ciencias experimentales como en ciencias sociales, dejan la beca antes de los cuatro años. Sólo el $10 \%$ de ellos lo hace porque les ha sobrado tiempo para hacer la tesis; las dos terceras partes dejan la beca por un contrato de profesor, una oportunidad que cuando surge no se puede desdeñar, aún a riesgo de que las obligaciones docentes impidan a partir de ese momento terminar la tesis. En este caso - muy frecuente - la tesis se convertirá de ejercicio de investigación a formalidad para progresar en la carrera docente. Comparto las consecuentes aprensiones de los autores de 'Sobran o faltan doctores' al respecto: cuando la tesis y las publicaciones son un requisito formal para progresar en al carrera docente, se sucumbe a veces a las presiones para evaluarlas al peso.

\section{ALGUNAS PROPUESTAS POLÍtiCAS}

Según hemos visto, hay datos que inducen a pensar que la elite académica española es menos brillante que la de otros países, $y$ hay pocos indicios, en cambio, que muevan al optimismo sobre la capacidad del sistema para formar mejores elites investigadoras. Quizás podría profundizarse el aprendizaje del inglés, quizás podría hacerse más general la formación en el Bachillerato y en la Universidad. Según proponen De Miguel y sus colegas, quizás se podrían convertir los cursos de doctorado en una enseñanza inequívocamente a tiempo completo, con un sistema de becas y ayudas más completo. Comparto esta propuesta suya, que en mi opinión debería concretarse separando los dos años necesarios para obtener el DEA, en los cuales las becas no irían ligadas al proyecto de tesis, sino sólo a las notas de los alumnos, y el período posterior de tesis doctoral. Quizás se podría intentar que los estudiantes de doctorado aprendan a investigar con profesores que realmente investigan, como reza la propuesta 4 del trabajo que aquí se glosa (p. 152), aunque dudo mucho de los procedimientos para separar el grano de la paja (no funcionan, desde luego, los del estilo de la ANECA). Y aunque no veo por qué razón iba a ser mejor concentrar la formación de doctores en algunas Universidades impidiendo a las otras impartir el grado (no se dejarían, y con razón), tampoco me opondría a una formación más imparcial de los tribunales de doctorado, dedicando, por ejemplo, unos pocos profesores a la sola tarea de evaluar tesis (tarea dura, que exigiría dedicación total y daría mucho poder a los elegidos). Todo esto y algunas cosas más seguramente no harían daño, pero es difícil que afectaran perceptiblemente a la minoría entre la que se reclutan los investigadores.

Teniendo una cantera pobre, con tendencia a empobrecerse aún más por el descenso demográfico, es particularmente grave que nuestro proceso de selección de investigadores sea claramente subóptimo. Por 'un lado está sin resolver el problema de la permanencia de los becarios en los OPIs, lo cual en la Universidad lleva a preferir el contrato de ayudante a la beca de investigación, y por 
otro se ha implantando un modelo de selección adversa a través de la contratación de profesores asociados que muchas veces se quedarán como investigadores. Sería de desear que se refuercen organizaciones de investigación independientes de la Universidad, tanto públicas como privadas, y que se abran vías de salida de los OPIs a la empresa pública y privada para los becarios excesivos y los investigadores fatigados. Para ello habría que mejorar notablemente la consideración laboral del título de doctor, correspondiendo a las Administraciones Públicas abrir camino; pero creo que hay un exceso de optimismo en esta previsión.

En cualquier caso, si se quiere realmente fomentar nuestra 'industria' de I+D hasta lograr que sea competitiva a nivel mundial, no basta con aumentar los presupuestos, racionalizar la distribución de los fondos y mejorar la gestión de los organismos investigadores, como se ha venido haciendo hasta ahora (Cruz y Sanz, 2001). Es además necesario competir por los mejores talentos en todo el mundo, estableciendo incentivos que atraigan a la $\mathrm{I}+\mathrm{D}$ a los mejores licenciados y mantengan en ella a los mejores doctores. Será preciso abrir nuestras universidades y organismos de investigación a la inmigración de cerebros. La investigación científica y técnica, más incluso que otras ramas de la producción, está hoy inserta en una división internacional del trabajo en la que no se puede competir con restricciones proteccionistas a la importación de mano de obra. Utilizando un símil futbolístico, podría decirse que si queremos ganar campeonatos mundiales no debemos competir como selecciones nacionales, sino como clubes que pugnan por alinear a las mejores estrellas. Es al fin y al cabo lo que hacen las famosas Universidades investigadoras de Estados Unidos, las mejores del mundo cuando juzga la calidad por el número de premios Nóbel. 


\section{REFERENCIAS}

Carabaña, Julio (1999) «Una propuesta para regular el ingreso y la promoción de los profesores universitarios». Tempora. Revista de historia y sociología de la Educación, segunda época, volumen 2:243-272.

Carabaña, Julio (2002) «El 'punto ciego' de la Ley de Universidades». Clavès de Razón Práctica, 119:32-41.

CARABAÑa, Julio (2004), «El sistema de enseñanza: participación y contenidos». En Laura Cruz (coord.), Prospectiva Social e investigación cientifica y tecnológica Madrid: FECYT

Cruz Castro, Laura y Luis Sanz Menéndez, (2001) «Autonomía y adaptación organizativa: los centros de investigación ante los cambios del entorno». Revista Española de Investigaciones Sociológicas, 95: 37-67;

FERNÁNDEZ ESQUINAS, MANUEL (2002) La formación de investigadores cientificos en España, Madrid:CIS.

LaRRAGa Rodríguez de Vera, Vicente E.(2003) «La pérdida de talentos científicos en España». DT 22/2003. Madrid: Fundación Alternativas.

MERTON, ROBERT K.(1987) «Three fragments from a sociologist's notebooks: Establishing the Phenomenon, Specified Ignorance and Strategic Research Materials», Annual Review of Sociology, 13:1-28.

Miguel, Jesús M. de, Bernabe Sarabia, Elizabeth G. Vaquera y Heizam Amirah, (2004) «¿Sobran o faltan doctores?. Empiria. Revista de metodología de ciencias sociales. 7 (115-155).

OCDE (2001) Connaissances et competences: des atouts pour la vie. Premiers résultats de PISA 2000. Enseignement et competénces. París: OCDE.

Pérez Díaz, Víctor y Juan Carlos RodríGuez (2003) La Educación General en España. Madrid: Fundación Santillana. 


\title{
RESUMEN.
}

El artículo «Sobran o faltan doctores « publicado en el número 7 de Empiria (De Miguel y otros, 2004) da una respuesta ambigua a la pregunta del título y propone diversas políticas. Estoy de acuerdo con su tesis principal: en España faltarían doctores si nuestro nivel de I+D fuera el que corresponde a la octava potencia económica del mundo. Difiero, sin embargo, en las cuestiones relativas a la eficiencia de los esttudios de doctorado y la desigualdad por sexo y región. Avanzo también una hipótesis sobre la debilidad de las elites investigadoras españolas y acabo con la propuesta de recurrir al mercado internacional de investigadores, de modo semejante a como recurrimos al de futbolistas.

\begin{abstract}
The paper «Sobran o faltan doctores», published in Empiria, 7 (De Miguel et alt. 2004) gives an ambiguous response to its main question and proposes various policy measures. I agree with its main thesis: it would be a lack of doctors in Spain if our I*D were to correspond with our economic level. I differ in secondary questions like the efficency of the doctarate programs or the inequality by sex or region. Moreover, I advance a hypothesis about the weakness of the spanish research elite and do finish with the proposal of looking for good researches in the international market, in the way we so much do for soccer.
\end{abstract}

